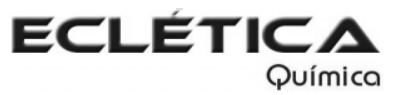

www.scielo.br/eq

Volume 31, número 1, 2006

\title{
Determinação de cromo (VI) por espectrometria de absorção atômica com chama após a extração e pré- concentração no ponto nuvem
}

\author{
A. Sussulini, M. A. Z. Arruda* \\ Universidade Estadual de Campinas (Unicamp), Instituto de Química, \\ Departamento de Química Analítica, Caixa Postal 6154, 13083-970, Campinas, São Paulo, Brasil \\ *Autor para correspondência.E-mail: zezzi@iqm.unicamp.br
}

Resumo: O presente trabalho reporta um método para a determinação de cromo (VI) por espectrometria de absorção atômica com chama (FAAS) após a extração e pré-concentração no ponto nuvem. $\mathrm{O}$ cromo (VI) é complexado com 1,5-difenilcarbazida (DFC) em meio ácido ( $\mathrm{pH}$ 2,0) e é extraído para um volume de fase rica de $25 \mu \mathrm{L}$, com o uso do surfactante Triton X-114. As variáveis que afetam a formação do ponto nuvem, tais como a concentração de surfactante $(0,1-1,0 \% \mathrm{~m} / \mathrm{v})$ e de complexante (0,01-0,80\% m/v), o tempo de complexação (0-60 min) e o efeito da adição do eletrólito $\mathrm{NaCl}$ (0-20\% $\mathrm{m} / \mathrm{v}$ ), são avaliadas. Sob condições otimizadas, são utilizados o Triton X-114 a 0,3\% m/v, a DFC a $0,05 \% \mathrm{~m} / \mathrm{v}$ e o $\mathrm{NaCl}$ a $10 \% \mathrm{~m} / \mathrm{v}$ para a extração do cromo (VI). Esse método fornece limites de detecção e quantificação de $0,4 \mu \mathrm{g} \mathrm{L}^{-1}$ e $1,5 \mu \mathrm{g} \mathrm{L}^{-1}$, respectivamente, e uma faixa linear de calibração de 5 a $500 \mu \mathrm{g} \mathrm{L}^{-1}$. O fator de pré-concentração obtido é igual a 27 e a eficiência de extração varia de 87 a $99,3 \%$.

Palavras-chave: extração por ponto nuvem; pré-concentração; espectrometria de absorção atômica com chama; cromo (VI).

\section{Introdução}

O cromo é um elemento de considerável importância ambiental e geológica e, no ambiente, ocorre principalmente como cromo (III) ou cromo (VI). As funções bioquímicas e os efeitos do cromo são dependentes de seu estado de oxidação, pois enquanto o cromo (VI) é tóxico por ser um agente carcinogênico, o cromo (III) é considerado um nutriente essencial para os humanos [1]. As maiores fontes de cromo (VI) são antropogênicas, originando-se principalmente de indústrias têxteis, de refinarias de petróleo e de galvanizações, e este é transferido ao ambiente por meio de emissões pelo ar ou pela água. Já o cromo (III) aparece difundido em níveis diminutos na natureza [1,2]. Devido ao fato de que os cromatos são amplamente empregados no tratamento de águas e que o cromo (VI) apresenta uma toxicidade muito maior que a do cromo (III), o maior interesse na especiação de cromo é focado na determinação de cromo (VI) [3].

A determinação direta de cromo em baixas concentrações não é possível de ser efetuada com sensibilidade suficiente, mesmo com o uso de métodos analíticos mais sofisticados, tais como a 
espectrometria de emissão óptica com fonte de plasma indutivamente acoplado (ICP OES) ou a espectrometria de absorção atômica com atomização eletrotérmica (ETAAS). Por isso, diversos métodos de extração e pré-concentração têm sido desenvolvidos, tais como a troca iônica, a extração em fase sólida, a coprecipitação, etc [4]. Recentemente, alguns métodos analíticos de extração e/ou pré-concentração mediados por surfactantes foram propostos como alternativas ao uso de solventes orgânicos [1,2,4-10].

Os surfactantes são importantes em química analítica por possuírem a característica de modificar algumas propriedades reacionais, com conseqüente melhoria em sensibilidade e/ou em seletividade analítica [11,12]. Quando uma solução de surfactante atinge uma determinada concentração (chamada concentração micelar crítica, CMC) suas moléculas se agregam espontaneamente, formando as micelas $[5,11]$.

A separação em duas fases isotrópicas, gerada a partir de sistemas micelares, é utilizada para extrair diversos tipos de analitos por meio do processo denominado extração no ponto nuvem (do inglês, cloud point extraction) e pode ser aplicada para a extração e/ou a pré-concentração de espécies inorgânicas (complexos metálicos), orgânicas e biomoléculas $[6,11,13]$.

A extração no ponto nuvem ocorre quando surfactantes não-iônicos, em solução aquosa e em quantidade acima da CMC, são aquecidos a uma determinada temperatura, conhecida como temperatura de ponto nuvem, na qual a solução torna-se turva. Esta turvação surge por haver um decréscimo da solubilidade do surfactante na água $[5,7,14]$. Acima da temperatura de ponto nuvem, são formadas duas fases e, após um certo tempo (ou após a centrifugação), duas fases líquidas são obtidas: uma que contém alta concentração de surfactante e os componentes extraídos da solução da amostra, denominada fase rica, e outra fase aquosa, que contém uma pequena concentração de surfactante, próxima à CMC, denominada fase pobre [11].

Além da versatilidade da extração no ponto nuvem como método de separação, outras vantagens são $[6,8,15]$ : i) a alta capacidade de pré-concentrar uma ampla variedade de analitos com boas eficiências de extração; ii) os surfac- tantes utilizados não são tóxicos e são menos perigosos que os solventes orgânicos comumente empregados nas extrações líquido-líquido por não serem voláteis ou inflamáveis, além do fato de que a quantidade e o custo dos surfactantes são menores; iii) a disposição final dos resíduos é facilitada, uma vez que estes podem ser incinerados; e iv) o fenômeno da separação de fases é reversível.

A principal limitação das extrações por ponto nuvem de íons metálicos é o baixo coeficiente de partição de muitas espécies neutras de quelatos metálicos, o que pode ser solucionado com o uso de ligantes altamente hidrofóbicos [6].

O emprego de sistemas micelares na espectrometria atômica é bastante vantajoso, pois apresentam a capacidade de aumentar a detecção por meio da manipulação das propriedades físicoquímicas da solução que contém a amostra: a eficiência da nebulização é melhorada devido à redução da tensão superficial da água, assim como a compatibilidade da fase aquosa por solventes orgânicos, porque os surfactantes apresentam a propriedade de estabilizar emulsões por meio da formação de um filme orientado na interface [16].

Assim sendo, uma metodologia baseada na extração e pré-concentração de cromo (VI) por ponto nuvem aliada à determinação por espectrometria de absorção atômica com chama (FAAS) como a apresentada neste trabalho, é bastante conveniente por ser uma alternativa simples, rápida, sensível e de baixo custo, em relação a outros métodos.

\section{Parte Experimental}

\section{Equipamentos}

Para o preparo das soluções foram utilizados uma balança analítica, modelo AE 200 (Mettler, Bedford, EUA) e um potenciômetro, modelo DM20 (Digimed, São Paulo, Brasil) para aferir o pH da solução tampão. Para o procedimento de extração por ponto nuvem foram utilizados uma placa aquecedora (Quimis, Diadema, Brasil) e um termômetro para preparar um banhomaria e uma centrífuga, modelo NT 811 (Nova Técnica, Piracicaba, Brasil), para acelerar o 
processo de separação de fases. A determinação de cromo (VI) foi efetuada em um espectrômetro de absorção atômica com chama, modelo AAnalyst 300, equipado com sistema de correção de fundo por lâmpada de deutério (Perkin-Elmer, Norwalk, EUA) e utilizando uma lâmpada de cátodo oco de cromo (Perkin-Elmer, Norwalk, EUA) como fonte de radiação primária, operada a um comprimento de onda de $357,9 \mathrm{~nm}$ e com uma fenda de $0,7 \mathrm{~nm}$.

\section{Reagentes, soluções e amostras}

Todas as soluções foram preparadas utilizando-se água desionizada (18,2 MW cm), obtida por um sistema de purificação Milli-Q (Millipore, Molsheim, França) e reagentes de grau analítico. As soluções estoque de cromo (VI) foram preparadas pela dissolução de quantidades apropriadas de $\mathrm{K}_{2} \mathrm{Cr}_{2} \mathrm{O}_{7}$ (Merck, Darmstadt, Alemanha) em água desionizada. A solução tampão a pH 2,0 foi preparada a partir de $25 \mathrm{~mL}$ de uma solução de $\mathrm{KCl}$ (Mallinckrodt, Xalostoc, México) a 0,2 mol L-1 , 6,5 mL de uma solução de $\mathrm{HCl}$ (Merck, Darmstadt, Alemanha) a 0,2 mol L1 e, então, diluída a $100 \mathrm{~mL}$ com água desionizada. As soluções de Triton X-114 (Aldrich, Milwaukee, EUA) e 1,5-difenilcarbazida (Merck, Darmstadt, Alemanha) foram preparadas pela dissolução das quantidades adequadas dos regentes em água desionizada. Para a curva analítica de calibração (5 a 500 _g L L-1), os volumes adequados da solução estoque de cromo (VI) foram diluídos em uma solução de $\mathrm{HNO}_{3}$ (Mallinckrodt, Xalostoc, México) a 0,1 $\mathrm{mol} \mathrm{L}^{-1} \mathrm{e}$ etanol (J.T. Baker, Phillipsburg, EUA) a 5\% v/v, na proporção 1:9 (essa solução é a mesma utilizada na diluição da fase rica). As amostras de água mineral utilizadas foram adquiridas em um supermercado local e a água de bebedouro foi coletada no Instituto de Química da Unicamp (Campinas, SP).

\section{Procedimento}

Em um tubo de ensaio, adiciona-se $1 \mathrm{~mL}$ de solução de cromo (VI), $1 \mathrm{~mL}$ de solução tampão $\mathrm{HCl}: \mathrm{KCl}$ 0,2 mol L-1, $1 \mathrm{~mL}$ de solução de 1,5-difenilcarbazida (DFC), e $7 \mathrm{~mL}$ de solução de Triton X-114 contendo NaCl. O tubo de ensaio é então vedado com Parafilme®, agitado e coloca- do em banho-maria a $85^{\circ} \mathrm{C}$ por 15 minutos, de modo que a separação de fases seja facilitada e ocorra mais rapidamente do que em temperatura ambiente. Após o resfriamento, o tubo é centrifugado por 10 minutos a $2500 \mathrm{rpm}$. A fase pobre é separada com o auxílio de uma pipeta. A fase rica é diluída com a solução de $\mathrm{HNO}_{3}$ :etanol descrita anteriormente e o volume é elevado a 1,8 mL em mini-frascos. Alíquotas de ambas as fases são conduzidas ao espectrômetro de absorção atômica para a determinação de cromo (VI).

\section{Resultados e discussão}

\section{Princípio do método}

A reação entre o cromato ou o dicromato e a DFC $\left(\mathrm{H}_{4} \mathrm{~L}\right)$, resulta na formação de um quelato de coloração violeta intensa $\left(\left[\mathrm{Cr}(\mathrm{HL})_{2}\right]^{+}\right)$. Esse quelato é constituído em cromo (III) e 1,5-difenilcarbazona $\left(\mathrm{H}_{2} \mathrm{~L}\right)$. A reação é representada pela equação [17]:

$2 \mathrm{CrO}_{4}^{2-}+3 \mathrm{H}_{4} \mathrm{~L}+8 \mathrm{H}^{+}=\left[\mathrm{Cr}(\mathrm{HL})_{2}\right]^{+}+$ $\mathrm{Cr}^{3+}+\mathrm{H}_{2} \mathrm{~L}+8 \mathrm{H}_{2} \mathrm{O}$. Essa reação ocorre em meio ácido, no qual o cromo está na forma de cromato e, assim, o cromo (VI) é reduzido e complexado pela DFC. O pH ideal para tal reação ocorrer é reportado na literatura como sendo igual a 2,0 [17] e, portanto, o estudo do efeito do $\mathrm{pH}$ na otimização do método não foi necessário.

\section{Efeito da concentração do surfactante}

A variação na eficiência de extração de cromo (VI) com a concentração do surfactante Triton X-114 foi analisada na faixa de 0,1 a 1,0\% $\mathrm{m} / \mathrm{v}$ e os resultados obtidos são mostrados na Tabela 1. O Triton X-114 foi o surfactante escolhido devido ao fato de possuir uma temperatura de ponto nuvem relativamente baixa e uma alta densidade da fase rica em surfactante, o que facilita a separação de fases por centrifugação, bem como pela ausência de grupos eletroativos em sua molécula [9]. Com base nos resultados obtidos, a concentração de Triton X-114 adotada para os estudos posteriores foi a de $0,3 \% \mathrm{~m} / \mathrm{v}$, por apresentar a melhor relação entre uma boa eficiência de extração e um volume da fase rica menor, de modo a favorecer a pré-concentração do analito. 
Tabela 1. Desempenho da extração no ponto nuvem com a variação da concentração de Triton X-114, utilizando soluções de $\mathrm{NaCl}$ a $10 \%$ m/v, DFC a $0,5 \% \mathrm{~m} / \mathrm{v}$ e cromo (VI) a $1 \mathrm{mg} \mathrm{L}^{-1}$. Determinações feitas em triplicata.

\begin{tabular}{ccc}
\hline $\begin{array}{c}\text { Concentração de } \\
\text { Triton X-114 / } \% \\
\mathrm{~m} / \mathrm{v}\end{array}$ & $\begin{array}{c}\text { Volume da fase } \\
\text { rica } / \mu \mathrm{L}\end{array}$ & $\begin{array}{c}\text { Eficiência da } \\
\text { extração }{ }^{\mathrm{a}} / \%\end{array}$ \\
\hline 0,1 & & \\
0,3 & 15 & $80,7 \pm 3,95$ \\
0,5 & 25 & $89,6 \pm 4,16$ \\
1,0 & 50 & $90,0 \pm 4,16$ \\
\hline
\end{tabular}

${ }^{\mathrm{a}}$ Proporção da concentração do metal na fase rica com aquela da solução original.

\section{Efeito da concentração do complexante}

$\mathrm{O}$ efeito da concentração do agente quelante, DFC, na extração e pré-concentração de cromo (VI) foi avaliado, na faixa de 0,01 a $0,80 \%$ $\mathrm{m} / \mathrm{v}$. O estudo não foi realizado para concentrações acima de $0,80 \% \mathrm{~m} / \mathrm{v}$ de $\mathrm{DFC}$, pois se formava um precipitado no meio. Segundo os resultados obtidos, mostrados na Figura 1, verifica-se que existe uma relação inversamente proporcional entre a concentração do complexante e a resposta analítica. Isso ocorre porque, quando a concentração do complexante é alta, as moléculas deste que não se ligaram ao metal, irão interagir com o surfactante e competir com as moléculas do complexo formado [18]. A concentração de DFC escolhida para os estudos seguintes foi a de $0,05 \% \mathrm{~m} / \mathrm{v}$, pois esta condição forneceu a maior resposta analítica, de acordo com a Figura 1.

\section{Efeito da adição de eletrólito}

Neste método de extração por ponto nuvem, a adição de eletrólito é imprescindível, pois, na sua ausência, quando a temperatura do sistema diminui e é feita a centrifugação, este voltava a ser monofásico, ou seja, o surfactante e o quelato metálico retornavam ao meio aquoso, resultando em uma grande perda na eficiência de extração. Assim, mantendo-se fixas as concen- trações de Triton X-114 e DFC otimizadas previamente e usando-se a concentração de cromo (VI) igual a $1 \mathrm{mg} \mathrm{L}^{-1}$, foi otimizada a concentração do eletrólito $\mathrm{NaCl}$, na faixa de 0 a $10 \% \mathrm{~m} / \mathrm{v}$. Os resultados obtidos são mostrados na Figura 2. Observou-se que a adição de eletrólito favoreceu a formação e a estabilização das micelas, obtendose, inclusive, valores de resposta analítica bem mais significativos do que na situação em que a força iônica não foi ajustada. O comportamento indica que, quando micelas mistas são formadas por surfactantes não-iônicos, elas ficam carregadas negativamente, sendo a valência do cátion um fator decisivo na alteração do ponto nuvem. $\mathrm{O}$ efeito dos eletrólitos sobre o ponto nuvem de surfactantes não-iônicos é explicado em termos dos efeitos salting-in e salting-out [10]. O efeito salting-out é diretamente relacionado a desorção de íons para as partes hidrofílicas das micelas, aumentando a inter-atração entre as micelas e, conseqüentemente, causando a precipitação das moléculas de surfactante [19]. Para concentrações de $\mathrm{NaCl}$ acima de $10 \% \mathrm{~m} / \mathrm{v}$, as fases ricas formadas ficaram na parte superior do sistema, indicando que pode ter ocorrido uma alteração na estrutura das micelas. A concentração de $\mathrm{NaCl}$ otimizada foi a de $10 \% \mathrm{~m} / \mathrm{v}$, por apresentar uma maior resposta analítica. 


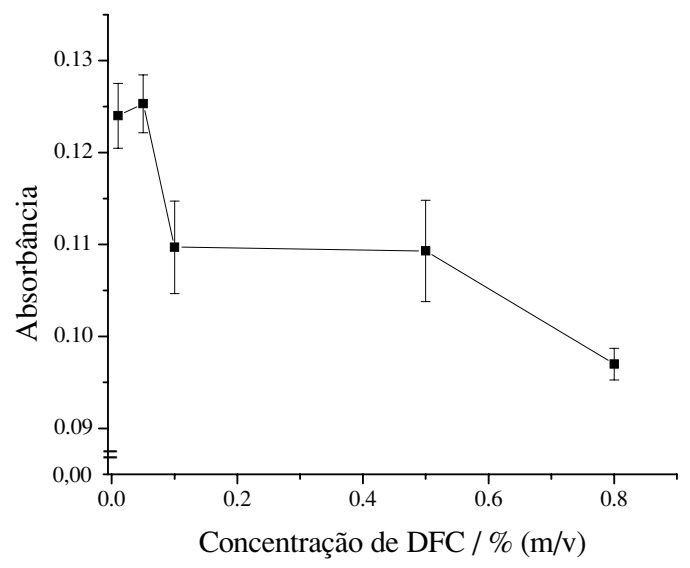

Figura 1. Efeito da concentração da DFC na absorbância. Determinações feitas em triplicata. Fixou-se a concentração de Triton X-114 em $0,3 \% \mathrm{~m} / \mathrm{v}$, a concentração de $\mathrm{NaCl}$ em $10 \% \mathrm{~m} / \mathrm{v}$ e a de cromo (VI) em 1,0 $\mathrm{mg} \mathrm{L}^{-1}$.

\section{Efeito do tempo de complexação}

As reações químicas que ocorrem na presença de agregados micelares geralmente apresentam fortes efeitos cinéticos, que podem ser explicados em termos da distribuição dos produtos e reagentes entre os agregados e a fase aquosa [18]. Assim sendo, realizou-se um estudo do efeito do tempo de complexação na pré-concentração e extração de cromo (VI). Foram mantidas fixas as condições otimizadas posteriormente e a concentração de cromo (VI) em $1 \mathrm{mg} \mathrm{L}^{-1}$. De acordo com os resultados obtidos, foi possível verificar que, nesse sistema, o tempo de complexação não afeta de modo significativo os parâmetros estudados (os valores de absorbância mantiveram-se constantes, em uma faixa de 0,126 a 0,129). Assim, por questão de praticidade, não houve necessidade de aplicar qualquer tempo de complexação.

Características analíticas do método e testes de adição e de recuperação

Nas condições otimizadas anteriormente (Triton X-114 a 0,3\% m/v, NaCl a $10 \% \mathrm{~m} / \mathrm{v}$, DFC a $0,05 \% \mathrm{~m} / \mathrm{v}$ e ausência de tempo de complexação), foram determinadas as características analíticas do método. Para isso, verificou-se a linearidade da resposta analítica em função da concentração de cromo (VI), obtendo-se os resultados

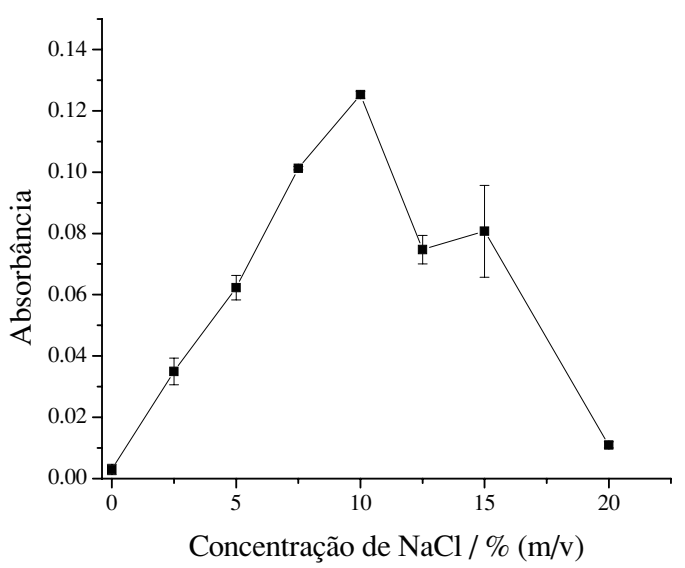

Figura 2. Variação da resposta analítica com a concentração de $\mathrm{NaCl}$. Determinações feitas em triplicata. Fixou-se a concentração de Triton X$114 \mathrm{em} 0,3 \% \mathrm{~m} / \mathrm{v}$, a de DFC em $0,05 \% \mathrm{~m} / \mathrm{v}$ e a de cromo (VI) em $1 \mathrm{mg} \mathrm{L}^{-1}$.

mostrados na Tabela 2. O fator de pré-concentração foi calculado como a proporção entre o coeficiente angular da curva de calibração com e sem o processo de pré-concentração [20,21]. O limite de detecção foi calculado como 3 vezes o desvio padrão obtido em 11 determinações do branco, dividido pela inclinação da curva de calibração. $\mathrm{O}$ limite de quantificação foi calculado como 10 vezes o desvio padrão obtido em 11 determinações do branco, dividido pela inclinação da curva de calibração, de acordo com a IUPAC [22].

De modo a validar o método proposto, foram realizados testes de adição e de recuperação, nos quais se partiu das condições otimizadas previamente e foram adicionados os íons cromo (VI) e cromo (III), em concentrações conhecidas e variadas. O cromo (III) foi somente empregado como possível concomitante. Por meio dos resultados obtidos, é possível afirmar que a recuperação do analito foi satisfatória (em média, $c a .87 \%$ ) e que a presença do íon cromo (III) não interfere significativamente na extração de cromo (VI).

\section{Análise de amostras de águas}

O método proposto neste trabalho foi aplicado na determinação de cromo (VI) em amostras reais, no caso, de águas minerais com e sem gás 
Tabela 2. Características analíticas dmétodo proposto.

\begin{tabular}{ll}
\hline Parâmetro & Valor obtido \\
\hline Relação entre o volume das fases $^{\mathrm{a}}$ & 0,0025 \\
Fator de pré-concentração $^{\mathrm{b}}$ & 27 \\
Eficiência de extração $^{\mathrm{c}} / \%$ & 95 \\
Limite de deteç̧ão / $\mu \mathrm{g} \mathrm{L}^{-1}$ & 0,4 \\
Limite de quantificação / $\mu \mathrm{g} \mathrm{L}^{-1}$ & 1,5 \\
Equação da regressão & $\mathrm{y}=2,03 \cdot 10^{-3} \mathrm{x}+7,21.10^{-2}$ \\
Coeficiente de correlação & 0,9996 \\
Faixa linear / $\mu \mathrm{g} \mathrm{L}^{-1}$ & $5-500$ \\
\hline
\end{tabular}

aProporção entre o volume final da fase rica e o da fase pobre.

${ }^{\mathrm{b}}$ Proporção entre o coeficiente angular da curva com analito sem pré-concentração com o da curva do analito pré-concentrado.

' Proporção da concentração do metal na fase rica com aquela da solução original.

Tabela 3. Determinação de cromo (VI) em amostras de águas, feitas em triplicata.

\begin{tabular}{cccc}
\hline Amostra & $\begin{array}{c}\mathrm{Cr}(\mathrm{VI}) \\
\text { adicionado } / \mu \mathrm{g} \mathrm{L}^{-} \text {determinado } / \mu \mathrm{g} \mathrm{L}{ }^{-}\end{array}$ & \\
& 1 & 1 & \% Recuperação $^{\mathrm{a}}$ \\
\hline Água mineral sem gás & 0 & $5,06 \pm 1,04$ & - \\
& 5 & $9,11 \pm 1,36$ & 90,6 \\
Água mineral com gás & 5 & $13,1 \pm 3,70$ & 87,0 \\
& 0 & $10,5 \pm 1,83$ & - \\
Água de bebedouro & 10 & $14,9 \pm 1,47$ & 96,1 \\
& 0 & $20,1 \pm 2,75$ & 98,0 \\
& 5 & $17,6 \pm 1,67$ & - \\
& 10 & $21,7 \pm 2,18$ & 96,0 \\
\hline
\end{tabular}

${ }^{\text {a }}$ Levando-se em conta somente as médias. 
e de bebedouro. Além da determinação das amostras em si, foi feita a determinação das amostras com adição de padrões de 5 e $10 \mathrm{mg} \mathrm{L}$ 1. Na Tabela 3, encontram-se os resultados obtidos, em triplicata. Em todos os casos, as concentrações de cromo (VI) nas amostras encontram-se abaixo do teor máximo em águas, permitido pelo CONAMA $\left(0,05 \mathrm{mg} \mathrm{L}^{-1}\right)$, de acordo com a Resolução 20/86 [23].

\section{Conclusões}

O método de extração e pré-concentração por ponto nuvem é uma importante aplicação do uso de surfactantes em química analítica. Tal método mostra-se como alternativa simples, sensível e de baixo custo em relação a outras técnicas de pré-concentração de metais em baixas concentrações. Para o cromo (VI), o método proposto neste trabalho forneceu um bom fator de préconcentração e uma eficiência de extração adequada, mostrando que além de aumentar a sensi- bilidade analítica para a determinação deste elemento, o método também pode ser aplicado na remoção de cromo (VI) de águas contaminadas, uma vez que esse metal possui características nocivas à saúde humana. O limite de detecção deste método mostrou-se melhor que aqueles observados em outros trabalhos propostos na literatura [4,5,9]. Outro fato importante foi a obtenção do ponto nuvem sem aquecimento, o que facilita os trabalhos de rotina.

\section{Agradecimentos}

Os autores agradecem à FAPESP (processo 03/00329-0) pela bolsa de estudo concedida para A.S., ao $\mathrm{CNPq}$ pela bolsa de produtividade de M.A.Z.A., bem como ao apoio financeiro do CNPq, CAPES e FAPESP.

Recebido em: 08/02/2006

Aceito em: 10/03/2006

A. Sussulini, M. A. Z. Arruda. Determination of chromium (VI) by flame atomic absorption spectrometry after cloud point extraction and preconcentration

\begin{abstract}
The present work reports a method for chromium (VI) determination by flame atomic absorption spectrometry (FAAS) after cloud point extraction and preconcentration. Chromium (VI) is complexed with 1,5-diphenylcarbazide (DFC) in acidic medium (pH 2.0) and it is extracted into $25 \mu \mathrm{L}$ of surfactant-rich phase containing Triton X-114. The variables that affect the cloud point formation, such as the surfactant $(0.1-1.0 \% \mathrm{~m} / \mathrm{v})$ and complexant $(0.01-0.80 \% \mathrm{~m} / \mathrm{v})$ concentrations, time of complexation (0-60 min), and effect of electrolyte $\mathrm{NaCl}(0-20 \% \mathrm{~m} / \mathrm{v})$ addition are evaluated. Under optimized conditions, $0.3 \% \mathrm{~m} / \mathrm{v}$ Triton X-114, $0.05 \% \mathrm{~m} / \mathrm{v}$ DFC and $10 \% \mathrm{~m} / \mathrm{v} \mathrm{NaCl}$ are used for chromium (VI) extraction. This method allows detection and quantification limits of $0.4 \mu \mathrm{g} \mathrm{L}^{-1}$ and $1.5 \mu \mathrm{g} \mathrm{L}$ ${ }^{1}$, respectively, and a linear calibration range from 5 to $500 \mu \mathrm{g} \mathrm{L}^{-1}$. The preconcentration factor obtained is 27 and the extraction efficiency achieved varies from 87 to $99.3 \%$.
\end{abstract}

Keywords: cloud point extraction; preconcentration; flame atomic absorption spectrometry; chromium (VI). 


\section{Referências}

[1] J. L. Manzoori, M. H. Sorouraddin, F. Shemiran, Anal. Lett. 29(11) (1996) 2007.

[2] C. Wang, D. F. Martin, B. B. Martin, J. Environ. Sci. Health A34(3) (1999) 705.

[3] A. Padarauskas, A. Judzentiene, E. Naujalis, V. Paliulionyte, J. Chromatogr. A 808(1-2) (1998) 193.

[4] F. Shemirani, S. D. Abkenar, A. A. Mirroshandel, M. S. Niasari, R. R. Kozania, Anal. Sci. 19(10) (2003) 1453.

[5] E. K. Paleologos, A. G. Vlessidis, M. I. Karayannis, N. P. Evmiridis, Anal. Chim. Acta 477(2) (2003) 223.

[6] E. K. Paleologos, C. D. Stalikas, S. M. Tzouwara-Karayanni, M. I. Karayannis, Anal. Chim. Acta 436(1) (2001) 49.

[7] D. L. Giokas, E. K. Paleologos, S M. Tzouwara-Karayanni, M. I. Karayannis, J. Anal. At. Spectrom. 16(5) (2001) 521.

[8] C. C. Nascentes, M. A. Z. Arruda, Talanta 61 (2003) 759.

[9] E. K. Paleologos, C. D. Stalikas, S. M. TzouwaraKarayanni, G. A. Pilidis, M. I. Karayannis, J. Anal. At. Spectrom. 15(3) (2000) 287.

[10] L. M. Coelho, M. A. Z. Arruda, Spectrochim. Acta B 60(5) (2005) 743.

[11] B. M. Cordero, J. L. P. Pavón, C. G. Pinto, M. E. F Laespada, Talanta 40(11) (1993) 1703.
[12] N. Maniasso, Quim. Nova 24(1) (2001) 87

[13] W. L. Hinze, E. Pramauro, Crit. Rev. Anal. Chem. 24(2) (1993) 133.

[14] C. D. Stalikas, Trends Anal. Chem. 21(5) (2002) 343.

[15] M. A. Bezerra, M. A. Z. Arruda, S. L. C. Ferreira, Appl. Spectrosc. Rev. 40(4) (2005) 269.

[16] A. Sanz-Medel, M. R. F. de la Campa, E. B. Gonzalez, M. L. Fernandez-Sanchez, Spectrochim. Acta B 54(2) (1999) 251

[17] G. J. Willems, N. M. Blaton, O. M. Peeters, C. J. de Ranter, Anal. Chim. Acta 88(2) (1977) 345.

[18] C. C. Nascentes, Emprego de diferentes estratégias para análises em larga escala: Screening, extração ultra-sônica e pré-concentração por ponto nuvem, Tese de Doutorado, Unicamp, Campinas (2002) 140.

[19] G. Komaromy-Hiller, N. Calkins, R. Wandruszka, Langmuir 12 (1996) 916.

[20] C. R. T. Tarley, M. A. Z. Arruda, Anal. Sci. 20 (2004) 961.

[21] S. L. C. Ferreira, V. A. Lemos, B. C. Moreira, A. C. S. Costa, R. E. Santelli, Anal. Chim. Acta 403 (2000) 259.

[22] Analytical Methods Committee, Analyst 11 (1987) 119.

[23] http://www.mma.gov.br/port/conama/res/res86/ res2086.html, acessado em 08 de janeiro de 2006. 